\title{
Rate redistribution in dispersive electron cooling
}

\author{
H. Zhao®* and M. Blaskiewicz \\ Brookhaven National Laboratory, Upton, New York 11973, USA
}

(Received 17 March 2021; accepted 20 July 2021; published 12 August 2021)

\begin{abstract}
It is known that dispersive coupling can be applied to realize three-dimensional cooling in stochastic cooling and laser cooling. In electron cooling, the transverse cooling rate is usually smaller than the longitudinal one, especially for high-energy beams. We find that dispersion can also be introduced into electron cooling (dispersive electron cooling) to redistribute the cooling rate between the longitudinal and transverse planes. In this paper, we present an analytical model to explain and estimate the rate redistribution in dispersive electron cooling, in which both ion dispersion and electron dispersion are included. It demonstrates that a small horizontal dispersion of the ions can enhance the horizontal cooling at the expense of longitudinal cooling, and the electron dispersion is also of benefit to this effect. We also find that this redistribution effect will be more significant when there is a large difference between the horizontal and longitudinal cooling rates, which will be of great significance for high-energy electron cooling in the future.
\end{abstract}

DOI: 10.1103/PhysRevAccelBeams.24.083502

\section{INTRODUCTION}

Since it was invented by Budker in 1967, electron cooling has been a powerful method to reduce the beam emittance and momentum spread in many heavy-ion accelerators [1,2]. In the future, high-energy electron cooling is needed for some facilities, such as electron ion colliders [3,4], which requires electron beams with at least tens of $\mathrm{MeV}$. So far, the highest energy electron cooling system with $4.3 \mathrm{MeV}$ electrons has been constructed and operated at Fermi National Accelerator Laboratory, Batavia, Illinois [5]. Recently, world's first rf-based electron cooler was successfully commissioned at Brookhaven National Laboratory (BNL), Upton, NY [6], opening the possibility of electron cooling at higher energies.

Usually, the difference in the strength of longitudinal and transverse friction force is less severe in low-energy electron coolers. Additionally, transverse angles can be compensated by a longitudinal magnetic field created by a reasonably simple solenoid [7]. For higher energies, the transverse momenta of the electron beam in the comoving frame will be much larger than the longitudinal momenta, $\left(p_{\perp} / m c=\gamma \theta_{\perp} \gg \sigma_{\gamma} / \gamma=p_{\|} / m c\right)$. This results in a very weak transverse cooling at high energy. Although a longitudinal magnetic field in the cooling section can increase the

\footnotetext{
*hezhao@bnl.gov

Published by the American Physical Society under the terms of the Creative Commons Attribution 4.0 International license. Further distribution of this work must maintain attribution to the author(s) and the published article's title, journal citation, and DOI.
}

cooling rates, the difference between transverse and longitudinal cooling rates is often still large. Therefore, some adjustments or compensations are needed to balance the threedimensional cooling rate in high-energy electron cooling.

It is known that longitudinal-horizontal coupling of ion motion due to dispersion has been successfully applied to stochastic cooling and laser cooling to realize cooling in three dimensions [8-10], but it has never been used in practice for electron cooling. However, dispersive electron cooling with ion dispersion has been studied and measured before $[11,12]$. Along with an ion dispersion function, these methods also require a horizontal gradient of the longitudinal cooling force, which is realized by a displacement of the electron beam position. For high energies, this gradient is due to a physical offset of the beams and the ensuing density gradient. For low energies, the transverse potential well generated by the space charge force supplies an additional velocity gradient.

In this paper, we demonstrate that ion dispersion alone can be used to redistribute the cooling rates between the transverse and longitudinal planes. No electron beam offset or electron beam dispersion is needed. Using a linearized friction force, we derive an analytical model to explain and estimate this redistribution effect. The ion dispersion can enhance the horizontal cooling at the expense of longitudinal cooling rate. We then add electron dispersion and show it can be of benefit. An analytical model shows good agreement with simulation results. This redistribution effect will be of particular value when there is a large difference between uncoupled horizontal and longitudinal cooling rates, as occurs for high-energy beams. 


\section{COOLING FORCE}

The nonmagnetized cooling force on an ion is given by [13]

$\boldsymbol{F}\left(\boldsymbol{r}, \boldsymbol{u}_{\boldsymbol{i}}\right)=-4 \pi m_{e} r_{e}^{2} Z^{2} c^{4} \int \ln \Lambda \frac{\boldsymbol{u}_{\boldsymbol{i}}-\boldsymbol{u}_{\boldsymbol{e}}}{\left|\boldsymbol{u}_{\boldsymbol{i}}-\boldsymbol{u}_{\boldsymbol{e}}\right|^{3}} f_{e}\left(\boldsymbol{r}, \boldsymbol{u}_{\boldsymbol{e}}\right) d \boldsymbol{u}_{\boldsymbol{e}}$,

where $Z$ is the atomic number of the ion, $m_{e}$ is the electron mass, $r_{e}$ is the classical electron radius, $c$ is the speed of light, $\ln \Lambda$ is the Coulomb logarithm, $\boldsymbol{u}_{\boldsymbol{i}}$ and $\boldsymbol{u}_{\boldsymbol{e}}$ are the velocities of ion and electron in the rest frame, respectively, and $f_{e}\left(\boldsymbol{r}, \boldsymbol{u}_{\boldsymbol{e}}\right)$ is the electron beam distribution in sixdimensional phase space. We assume that the coordinates and velocity of electrons are not correlated and the beam distribution remains unchanged along the cooling section, the electron beam distribution can be written as $f_{e}\left(\boldsymbol{r}, \boldsymbol{u}_{\boldsymbol{e}}\right)=$ $n_{e}(\boldsymbol{r}) f_{v_{e}}\left(\boldsymbol{u}_{\boldsymbol{e}}\right)$, where $n_{e}$ and $f_{v e}$ are the number density and velocity distribution of electrons in the rest frame. Then, the integral in Eq. (1) depends only on the velocity distribution. Because the dispersion on the ion beam will only introduce the coupling between the horizontal position and the energy spread of ions, this integral will not be changed by the ion dispersion. If we take the Coulomb logarithm outside the integral in Eq. (1) what is left has the form of an electrostatic field. We take an isotropic electron velocity distribution with rms spread $\sigma_{v e}$ and a Gaussian distribution. The cooling force can be calculated analytically $[14,15]$

$$
\begin{aligned}
\boldsymbol{F} & =K \int_{0}^{\infty} \frac{\boldsymbol{u}_{\boldsymbol{i}}}{\left(\sigma_{v e}^{2}+\lambda\right)^{5 / 2}} \exp \left[-\frac{\boldsymbol{u}_{\boldsymbol{i}}{ }^{2}}{2\left(\sigma_{v e}^{2}+\lambda\right)}\right] d \lambda \\
& =K\left[\frac{\sqrt{2 \pi}}{\boldsymbol{u}_{\boldsymbol{i}}{ }^{2}} \operatorname{erf}\left(\frac{\boldsymbol{u}_{\boldsymbol{i}}}{\sqrt{2 \sigma_{v e}^{2}}}\right)-\frac{2}{\sigma_{v e} \boldsymbol{u}_{i}} \exp \left(-\frac{\boldsymbol{u}_{\boldsymbol{i}}^{2}}{2 \sigma_{v e}^{2}}\right)\right],
\end{aligned}
$$

where $K=-2 \sqrt{2 \pi} m_{e} r_{e}^{2} Z^{2} c^{4} \ln \Lambda n_{e}(\boldsymbol{r})$ and erf is the error function. We only work on the leading order of the velocity so that the cooling force can be expanded as

$$
\boldsymbol{F} \simeq K \frac{2 \boldsymbol{u}_{i}}{3 \sigma_{v e}^{3}}, \quad\left(\left|\boldsymbol{u}_{i}\right|<\sigma_{v e}\right) .
$$

We see that this linear cooling force is the same as the result in Ref. [13] for small $\boldsymbol{u}_{\boldsymbol{i}}$. Based on this approximation, we get the change of $\boldsymbol{u}_{\boldsymbol{i}}$ at the location $\boldsymbol{r}$ is

$\Delta \boldsymbol{u}_{\boldsymbol{i}}=-\frac{4 \sqrt{2 \pi} Z^{2} r_{e}^{2} c^{3} m_{e} \ln \Lambda n_{e}(\boldsymbol{r}) l}{3 \beta \gamma \sigma_{v_{e}}^{3} m_{i}} \boldsymbol{u}_{i}=-C n_{e}(\boldsymbol{r}) \boldsymbol{u}_{\boldsymbol{i}}$,

where $l$ is the length of the cooling section. We see that the cooling effect can be simply described by the coefficient $C$ and the number density of electron beam $n_{e}(\boldsymbol{r})$. This expression also applies to the electron beam with spherically symmetric velocity distribution. In the following, we will use Eq. (4) to build the analytical model of dispersive cooling. We will focus on the fundamental effect and apply cooling in the form of a thin kick.

\section{ION DISPERSION}

Now, we consider an ion with the initial momentum offset $\delta_{0}$ and horizontal dispersion $D_{i}$, the initial horizontal emittance is

$$
\epsilon_{x 0}=\left(x_{0}-D_{i} \delta_{0}\right)^{2} / 2 \beta_{x}+\beta_{x} x_{0}^{\prime 2} / 2 .
$$

After passing the cooling section,

$$
\epsilon_{x 1}=\left(x_{1}-D_{i} \delta_{1}\right)^{2} / 2 \beta_{x}+\beta_{x} x_{1}^{\prime 2} / 2 .
$$

Here, we did not consider the betatron motion along the cooling section. Since the cooling effect is quite small for a single pass, we have $x_{0}=x_{1}=x, x_{1}^{\prime}-x_{0}^{\prime}=-C n_{e}(\boldsymbol{r}) x_{0}^{\prime}$, and $\delta_{1}-\delta_{0}=-C n_{e}(\boldsymbol{r}) \delta_{0}$. Then the changes of emittance and momentum spread due to cooling for a single particle are

$\Delta \epsilon_{x}=-C n_{e}\left(\beta_{x} x^{\prime 2}-\frac{D_{i} \delta x_{\beta}}{\beta_{x}}\right)+C^{2} n_{e}^{2}\left(\frac{D_{i}^{2} \delta^{2}}{2 \beta_{x}}+\frac{\beta_{x} x^{\prime 2}}{2}\right)$,

$\Delta \delta^{2}=-2 C n_{e} \delta^{2}+C^{2} n_{e}^{2} \delta^{2}$,

$\Delta \epsilon_{y}=-C n_{e} \beta_{y} y^{\prime 2}$,

where $x_{\beta}=x-D_{i} \delta$ is the betatron amplitude. All the variables on the right-hand side correspond to the distribution before the cooling section. The local number density of electron beam (Gaussian) at the position $(x, y, s)=$ $\left(x_{\beta}+D_{i} \delta, y_{\beta}, s\right)$ is given by

$$
\begin{aligned}
n_{e}(x, y, s)= & \frac{N_{e 0}}{(2 \pi)^{3 / 2} \sigma_{e x} \sigma_{e y} \sigma_{e s}} \\
& \cdot \exp \left[-\frac{\left(x_{\beta}+D_{i} \delta\right)^{2}}{2 \sigma_{e x}^{2}}-\frac{y_{\beta}{ }^{2}}{2 \sigma_{e y}^{2}}-\frac{s^{2}}{2 \sigma_{e s}^{2}}\right],
\end{aligned}
$$

where $N_{e 0}$ is the total number of electrons. Now the problem is to calculate the average changes of the ion beam, which correspond to the cooling rates.

We assume the ion beam also has a Gaussian distribution, the joint probability density function is

$$
\begin{aligned}
P_{i}= & \frac{1}{(2 \pi)^{3} \sigma_{i x} \sigma_{i y} \sigma_{i s} \sigma_{i x^{\prime}} \sigma_{i y^{\prime}} \delta_{i p}} \\
& \cdot \exp \left(-\frac{x_{\beta}^{2}}{2 \sigma_{i x}^{2}}-\frac{y_{\beta}^{2}}{2 \sigma_{i y}^{2}}-\frac{s^{2}}{2 \sigma_{i s}^{2}}-\frac{x^{\prime 2}}{2 \sigma_{i x^{\prime}}^{2}}-\frac{y^{\prime 2}}{2 \sigma_{i y^{\prime}}^{2}}-\frac{\delta^{2}}{2 \delta_{i p}^{2}}\right),
\end{aligned}
$$

where $\sigma_{i x}, \sigma_{i y}, \sigma_{i s}, \sigma_{i x^{\prime}}, \sigma_{i y^{\prime}}$, and $\delta_{i p}$ are the standard deviation of the beam distribution. The derivation of the 

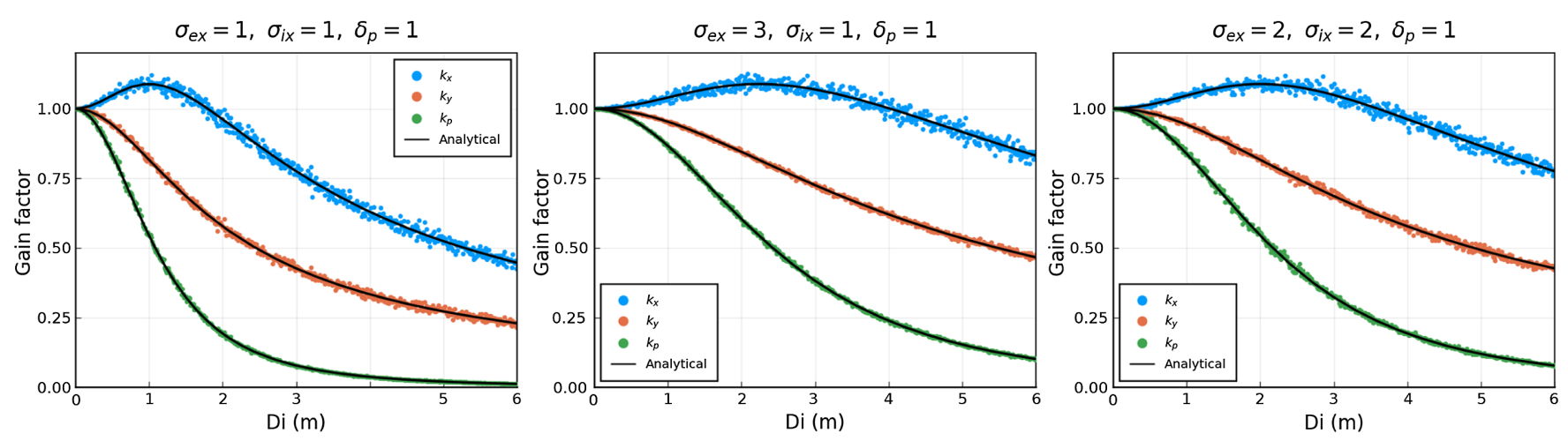

FIG. 1. The Monte Carlo and analytical results of the gain factor under different beam conditions.

average changes is tedious, and we here only give an example of the derivation on the horizontal emittance. Consider the first line of Eq. (7). To first order, we have

$$
\left\langle\Delta \epsilon_{x 1}\right\rangle=-C \beta_{x}\left\langle n_{e} x^{\prime 2}\right\rangle+\frac{C D_{i}}{\beta_{x}}\left\langle n_{e} \delta x_{\beta}\right\rangle,
$$

where \langle\rangle denotes averaging over the ion phase space. Based on Ref. [16], we know

$$
\begin{aligned}
\left\langle n_{e} x^{\prime 2}\right\rangle & =\int_{-\infty}^{\infty} n_{e} x^{\prime 2} P_{i} d x_{\beta} d y_{\beta} d s d x^{\prime} d y^{\prime} d \delta, \\
\left\langle n_{e} \delta x_{\beta}\right\rangle & =\int_{-\infty}^{\infty} n_{e} \delta x_{\beta} P_{i} d x_{\beta} d y_{\beta} d s d x^{\prime} d y^{\prime} d \delta .
\end{aligned}
$$

The relevant integrals are straightforward and are relegated to the Appendix. The details for the second-order are also given in the Appendix. It shows that the second-order term can be ignored. Based on these calculation, we finally get the dispersive cooling rate

$$
\begin{aligned}
& \lambda_{x}=\frac{\left\langle\Delta \epsilon_{x}\right\rangle}{\epsilon_{i x, \mathrm{rms}}}=-\hat{n}_{e 0}\left(C+\frac{C D_{i}^{2} \delta_{i p}^{2}}{\sigma_{e x}^{2}+\sigma_{i x}^{2}+D_{i}^{2} \delta_{i p}^{2}}\right), \\
& \lambda_{p}=\frac{\langle\delta\rangle}{\delta_{i p}}=-\hat{n}_{e 0}\left(C-\frac{C D_{i}^{2} \delta_{i p}^{2}}{\sigma_{e x}^{2}+\sigma_{i x}^{2}+D_{i}^{2} \delta_{i p}^{2}}\right), \text { and } \\
& \lambda_{y}=\frac{\left\langle\Delta \epsilon_{y}\right\rangle}{\epsilon_{i y, \mathrm{rms}}}=-\hat{n}_{e 0} C,
\end{aligned}
$$

where $\epsilon_{\mathrm{rms}}$ is the rms beam emittance with $\epsilon_{i x, i y, \text { rms }}=$ $\sigma_{i x, i y}^{2} / \beta_{x, y}=\sigma_{i x^{\prime}, i y^{\prime}}^{2} \beta_{x, y}$, and

$$
\hat{n}_{e 0}=\frac{(2 \pi)^{-3 / 2} N_{e 0}}{\sqrt{\left(\sigma_{e y}^{2}+\sigma_{i y}^{2}\right)\left(\sigma_{e s}^{2}+\sigma_{i s}^{2}\right)\left(\sigma_{e x}^{2}+\sigma_{i x}^{2}+D_{i}^{2} \delta_{i p}^{2}\right)}} .
$$

It is clear that the transverse and longitudinal cooling rates are modulated by dispersion. We see that the dispersion on the one hand results in the reduction of the cooling rate in all three dimensions, which is due to the density change of ion beam in $\hat{n}_{e 0}$. On the other hand, the coupling effect of dispersion transfers the cooling rate from longitudinal direction to horizontal direction. To estimate the net effect of dispersion on cooling rate, we define the gain factor as the ratio of the cooling rates with and without dispersion

$$
\begin{aligned}
& k_{x}=\frac{\lambda_{x}}{\lambda_{x, D_{i}=0}}=\frac{\sigma_{e x}^{2}+\sigma_{i x}^{2}+2 D_{i}^{2} \delta_{i p}^{2}}{\left(\sigma_{e x}^{2}+\sigma_{i x}^{2}+D_{i}^{2} \delta_{i p}^{2}\right)^{3 / 2}} \sqrt{\sigma_{e x}^{2}+\sigma_{i x}^{2}}, \\
& k_{p}=\frac{\lambda_{p}}{\lambda_{p, D_{i}=0}}=\frac{\left(\sigma_{e x}^{2}+\sigma_{i x}^{2}\right)^{3 / 2}}{\left(\sigma_{e x}^{2}+\sigma_{i x}^{2}+D_{i}^{2} \delta_{i p}^{2}\right)^{3 / 2}}, \text { and } \\
& k_{y}=\frac{\lambda_{y}}{\lambda_{y, D_{i}=0}}=\frac{\left(\sigma_{e x}^{2}+\sigma_{i x}^{2}\right)^{1 / 2}}{\left(\sigma_{e x}^{2}+\sigma_{i x}^{2}+D_{i}^{2} \delta_{i p}^{2}\right)^{1 / 2}} .
\end{aligned}
$$

Its obvious that $k_{p} \leq k_{y} \leq 1$, which means longitudinal and vertical cooling will always be weakened. The dependence of $k_{x}$ on dispersion is not obvious from Eq. (13). We calculate the gain factors under several conditions using arbitrary parameters. The result is shown in Fig. 1. It shows that for a small dispersion, the horizontal cooling rate is increased, which is mainly from longitudinal direction due to the coupling effect. When ion dispersion is large, both transverse and longitudinal cooling will be reduced because the ion beam size will be larger than the electron beam. Based on Eq. (7), we also apply the Monte Carlo method to check the gain factors, in which a group of particles with Gaussian distribution are generated and cooled, and the gain factors and its dependence on dispersion are calculated. As shown in Fig. 1, the Monte-Carlo method gives the same result as the analytical model. The condition for the maximum of $k_{x}$ is $2 D_{i}^{2} \delta_{i p}^{2}=\sigma_{e x}^{2}+\sigma_{i x}^{2}$, at which the horizontal cooling rate is maximized by dispersion. The maximum value of $k_{x}$ is $k_{x, \max }=\frac{4}{3} \sqrt{\frac{2}{3}}$, which is independent of beam parameters.

In above, we assume the coefficients of cooling force in three dimensions are the same, which makes the 



FIG. 2. The Monte Carlo and analytical results of the gain factor with $C_{p} / C_{x}=3$.

enhancement of horizontal cooling not obvious (up to $8.8 \%$ ). At high energy, the horizontal temperature of electron beam in the rest frame is usually higher than the longitudinal temperature, which causes a big difference between the horizontal and longitudinal cooling. We still consider the linear cooling force but different strength in horizontal and longitudinal planes: $\Delta \boldsymbol{u}_{x, p}=-C_{x, p} n_{e} \boldsymbol{u}_{x, p}$. Using the same method in the Appendix, we get the updated cooling rates:

$$
\begin{aligned}
& \lambda_{x}=-\hat{n}_{e 0}\left(C_{x}+\frac{C_{p} D_{i}^{2} \delta_{i p}^{2}}{\sigma_{e x}^{2}+\sigma_{i x}^{2}+D_{i}^{2} \delta_{i p}^{2}}\right), \\
& \lambda_{p}=-\hat{n}_{e 0}\left(C_{p}-\frac{C_{p} D_{i}^{2} \delta_{i p}^{2}}{\sigma_{e x}^{2}+\sigma_{i x}^{2}+D_{i}^{2} \delta_{i p}^{2}}\right) .
\end{aligned}
$$

and the gain factors

$k_{x}=\frac{\sigma_{e x}^{2}+\sigma_{i x}^{2}+\left(1+C_{p} / C_{x}\right) D_{i}^{2} \delta_{i p}^{2}}{\left(\sigma_{e x}^{2}+\sigma_{i x}^{2}+D_{i}^{2} \delta_{i p}^{2}\right)^{3 / 2}} \sqrt{\sigma_{e x}^{2}+\sigma_{i x}^{2}} \quad$ and

$k_{p}=\frac{\left(\sigma_{e x}^{2}+\sigma_{i x}^{2}\right)^{3 / 2}}{\left(\sigma_{e x}^{2}+\sigma_{i x}^{2}+D_{i}^{2} \delta_{i p}^{2}\right)^{3 / 2}}$.

We see that the redistribution effect is directly determined by the ratio of the cooling coefficients $C_{p} / C_{x}$. So, this effect would be much stronger if $C_{p}$ is larger than $C_{x}$, which is exactly corresponds to high-energy beam cooling. The maximum value of $k_{x}$ is $k_{x, \max }=$ $\frac{2}{3 \sqrt{3}}\left(1+\frac{C_{p}}{C_{x}}\right) \sqrt{1+\frac{C_{x}}{C_{p}}}$. The calculation with $C_{p} / C_{x}=3$ is shown in Fig. 2. It shows that the increase of horizontal cooling rate is significant (up to $\sim 80 \%$ ).

\section{ELECTRON DISPERSION}

The effect of electron dispersion on cooling was well described in Ref. [17]. Here, we include the contribution of the electron beam density. Considering the electron dispersion $D_{e}$, horizontal offset $x_{\text {off }}$ and energy offset $\delta_{\text {off }}$, the electron beam with Gaussian distribution can be described by $f_{e} \propto f_{e x} f_{e y} f_{e z}$ where [17]

$$
\begin{aligned}
& f_{e x}=\exp \left[-\frac{1}{2 \epsilon_{e x}}\left(\frac{1+\alpha_{e x}^{2}}{\beta_{e x}} \hat{x}^{2}+2 \alpha_{e x} \hat{x} x^{\prime}+\beta_{e x} x^{\prime 2}\right)\right], \\
& f_{e y}=\exp \left[-\frac{1}{2 \epsilon_{e y}}\left(\frac{1+\alpha_{e y}^{2}}{\beta_{e y}} y^{2}+2 \alpha_{e y} y y^{\prime}+\beta_{e y} y^{\prime 2}\right)\right], \\
& f_{e z}=\exp \left[-\frac{\left(\delta-\delta_{\text {off }}\right)^{2}}{2 \sigma_{e p}^{2}}-\frac{s^{2}}{2 \sigma_{e s}^{2}}\right],
\end{aligned}
$$

where $\hat{x}=x-x_{\mathrm{off}}-D_{e} \delta, \alpha_{e}$ and $\beta_{e}$ are the Twiss parameters. Note that $x^{\prime}$ and $y^{\prime}$ are the coordinates in the lab frame. In order to simplify the calculation of cooling force, we still try to write the beam distribution into the form: $f_{e}\left(\boldsymbol{r}, \boldsymbol{u}_{\boldsymbol{e}}\right)=n_{e}(\boldsymbol{r}) f_{v_{e}}\left(\boldsymbol{u}_{\boldsymbol{e}}\right)$. Based on Eq. (16), the density in the rest frame can be expressed as

$n_{e}=n_{e 0} \exp \left[-\frac{\left(x-x_{\mathrm{off}}-D_{e} \delta_{\mathrm{off}}\right)^{2}}{2\left(\epsilon_{e x} \beta_{e x}+D_{e}^{2} \delta_{e p}^{2}\right)}-\frac{y^{2}}{2 \epsilon_{e y} \beta_{e y}}-\frac{s^{2}}{2 \sigma_{e s}^{2}}\right]$

and the velocity distribution of electron beam in the rest frame is

$$
\begin{aligned}
f_{v_{e}}= & \frac{(2 \pi)^{-3 / 2}}{\sigma_{1} \sigma_{2} \sigma_{3} \sqrt{1-\rho^{2}}} \exp \left\{-\frac{\left(u_{y}-\bar{u}_{y}\right)^{2}}{2 \sigma_{2}^{2}}-\frac{1}{2\left(1-\rho^{2}\right)} .\right. \\
& \left.\times\left[\frac{\left(u_{x}-\bar{u}_{x}\right)^{2}}{\sigma_{1}^{2}}+\frac{\left(u_{p}-\bar{u}_{p}\right)^{2}}{\sigma_{3}^{2}}-2 \rho \frac{\left(u_{x}-\bar{u}_{x}\right)\left(u_{p}-\bar{u}_{p}\right)}{\sigma_{1} \sigma_{3}}\right]\right\},
\end{aligned}
$$

where $u_{x}=\beta \gamma x^{\prime}, u_{y}=\beta \gamma y^{\prime}$, and $u_{z}=\Delta p / p$ are the coordinates in the rest frame. The various parameters are given by 


$$
\begin{aligned}
\bar{u}_{x} & =-\frac{\gamma \alpha_{e x} \epsilon_{e x}\left(x-x_{\mathrm{off}}-D_{e} \delta_{\mathrm{off}}\right)}{\epsilon_{e x} \beta_{e x}+D_{e}^{2} \delta_{e p}^{2}}, \\
\bar{u}_{y} & =-\frac{\gamma \alpha_{e y} y}{\beta_{e y}}, \\
\bar{u}_{p} & =\frac{D_{e} \delta_{e p}^{2}\left(x-x_{\mathrm{off}}\right)+\epsilon_{e x} \beta_{e x} \delta_{\mathrm{off}}}{\epsilon_{e x} \beta_{e x}+D_{e}^{2} \delta_{e p}^{2}}, \\
\sigma_{1}^{2} & =\frac{\epsilon_{e x} \gamma^{2}}{\beta_{e x}}\left(1+\frac{\alpha_{e x}^{2} D_{e}^{2} \delta_{e p}^{2}}{\epsilon_{e x} \beta_{e x}+D_{e}^{2} \delta_{e p}^{2}}\right), \\
\sigma_{2}^{2} & =\frac{\epsilon_{e y} \gamma^{2}}{\beta_{e y}}, \\
\sigma_{3}^{2} & =\frac{\delta_{e p}^{2} \epsilon_{e x} \beta_{e x}}{\epsilon_{e x} \beta_{e x}+D_{e}^{2} \delta_{e p}^{2}}, \\
\rho & =\frac{\alpha_{e x} D_{e} \delta_{e p}}{\sqrt{\epsilon_{e x} \beta_{e x}+D_{e}^{2} \delta_{e p}^{2}\left(1+\alpha_{e x}^{2}\right)}} .
\end{aligned}
$$

The equations above for cooling force calculation are good for computer work but difficult analytically. To simplify the model, we still consider the linear cooling force and assume there are no phase shift and beam offsets $\left(\alpha_{e}=0, x_{\text {off }}=0\right.$, and $\left.\delta_{\text {off }}=0\right)$, then the cooling effect on a single particle can be approximately described as

$$
\Delta \boldsymbol{u}_{\boldsymbol{p}}=g C_{p} n_{e}\left(k x-\boldsymbol{u}_{\boldsymbol{p}}\right) \quad \Delta \boldsymbol{u}_{\boldsymbol{x}}=-C_{x} n_{e} \boldsymbol{u}_{\boldsymbol{x}},
$$

where $g=\frac{\sqrt{\sigma_{e x}^{2}+D_{e}^{2} \delta_{e p}^{2}}}{\sigma_{e x}}$ and $k=\frac{D_{e} \delta_{e p}^{2}}{\sigma_{e x}^{2}+D_{e}^{2} \delta_{e p}^{2}}$ are the coupling factors due to electron dispersion. Considering this approximation and ignoring high-order terms, Eq. (7) becomes

$\Delta \epsilon_{x}=-C_{x} n_{e} \beta_{x} x^{\prime 2}-g C_{p} n_{e}\left[\frac{k D_{i} x_{\beta}^{2}}{\beta_{x}}+\frac{D_{i}\left(1-k D_{i}\right) \delta x_{\beta}}{\beta_{x}}\right]$ and $\Delta \delta^{2}=-2 g C_{p} n_{e}\left[\left(1-k D_{i}\right) \delta^{2}-k \delta x_{\beta}\right]$.

Using the same method in the Appendix, the cooling rates with both electron dispersion and ion dispersion are

$$
\begin{aligned}
& \lambda_{x}=-\hat{n}_{e 0}\left[C_{x}+\frac{g C_{p}\left(D_{i}^{2} \delta_{i p}^{2}+D_{i} D_{e} \delta_{e p}^{2}\right)}{\sigma_{e x}^{2}+\sigma_{i x}^{2}+D_{i}^{2} \delta_{i p}^{2}+D_{e}^{2} \delta_{e p}^{2}}\right], \\
& \lambda_{p}=-\hat{n}_{e 0}\left[g C_{p}-\frac{g C_{p}\left(D_{i}^{2} \delta_{i p}^{2}+D_{i} D_{e} \delta_{e p}^{2}\right)}{\sigma_{e x}^{2}+\sigma_{i x}^{2}+D_{i}^{2} \delta_{i p}^{2}+D_{e}^{2} \delta_{e p}^{2}}\right],
\end{aligned}
$$

and $\lambda_{y}=-\hat{n}_{e 0} C_{y}$, where

$$
\hat{n}_{e 0}=\frac{N_{e 0}}{(2 \pi)^{3 / 2} \sqrt{L\left(\sigma_{e y}^{2}+\sigma_{i y}^{2}\right)\left(\sigma_{e s}^{2}+\sigma_{i s}^{2}\right)}},
$$

and $L=\sigma_{e x}^{2}+\sigma_{i x}^{2}+D_{i}^{2} \delta_{i p}^{2}+D_{e}^{2} \delta_{e p}^{2}$. The gain factors as a function of $D_{i}$ and $D_{e}$ are given by

$k_{x}=\left(L+\frac{g C_{p}}{C_{x}} D_{i}^{2} \delta_{i p}^{2}+\frac{g C_{p}}{C_{x}} D_{i} D_{e} \delta_{e p}^{2}\right) \sqrt{\frac{\sigma_{e x}^{2}+\sigma_{i x}^{2}}{L^{3}}}$ and

$k_{p}=g\left(L-D_{i}^{2} \delta_{i p}^{2}-D_{i} D_{e} \delta_{e p}^{2}\right) \sqrt{\frac{\sigma_{e x}^{2}+\sigma_{i x}^{2}}{L^{3}}}$

From Eqs. (21) and (22), we see that electron dispersion also contributes to the redistribution effect, but only when there is ion dispersion. A simple explanation for this is that electron dispersion reduces the local longitudinal energy spread $\left[\sigma_{3}\right.$ in Eq. (18)], thereby increasing the longitudinal cooling force. At the same time, with the coupling effect due to ion dispersion, the cooling rate redistribution effect is strengthened. The dependence of the gain factor on electron dispersion and ion dispersion is shown in Fig. 3, in which


FIG. 3. Dependence of the gain factors on electron dispersion and ion dispersion $\left(\sigma_{e x}=\sigma_{i x}=\delta_{e p}=\delta_{i p}=1, C_{p} / C_{x}=3\right)$. 
TABLE I. Beam parameters in simulation.

\begin{tabular}{lcc}
\hline \hline & Electron & Proton \\
\hline Energy $(\mathrm{GeV})$ & 0.15 & 275 \\
Relativistic factor $\gamma$ & 293.2 & 293.2 \\
Number of particles per bunch & $3 \times 10^{11}$ & $6.9 \times 10^{10}$ \\
RMS emittance $x / y(\mathrm{~nm})$ & $21 / 18$ & $9.6 / 1.5$ \\
$\mathrm{RMS} d p / p$ & $8.9 \times 10^{-4}$ & $6.6 \times 10^{-4}$ \\
$\mathrm{RMS}$ bunch length $(\mathrm{m})$ & 0.12 & 0.06 \\
$\beta_{x}^{*} / \beta_{y}^{*}$ function & $153 / 275$ & $100 / 100$ \\
$\sigma_{x} / \sigma_{y}(\mathrm{~mm})$ & $1.8 / 2.2$ & $1.0 / 0.4$ \\
$\sigma_{x^{\prime}} / \sigma_{y^{\prime}}\left(\times 10^{-5}\right)$ & $1.2 / 0.8$ & $1.0 / 0.4$ \\
Longitudinal temperature $(\mathrm{eV})$ & 0.41 & 410 \\
Transverse temperature $x / y(\mathrm{eV})$ & $6.0 / 2.9$ & $7760 / 1210$ \\
$L_{\text {cool }}(\mathrm{m})$ & 170 & 170 \\
\hline \hline
\end{tabular}

arbitrary parameters are used $\left(\sigma_{e x}=\sigma_{i x}=\delta_{e p}=\delta_{i p}=1\right.$, $\left.C_{p} / C_{x}=3\right)$. It shows that the value of $k_{x}$ can be continuously increased by electron dispersion and ion dispersion.

However, the assumption of cooling force in Eq. (19) is simple since we only considered the coupling effect in longitudinal direction. In fact, the coefficient $C_{x}$ is also related to the electron dispersion, which is not included in the model.

\section{SIMULATION}

In Secs. III and IV, we present an analytical model to study the cooling redistribution effect caused by dispersion, in which the nonlinear cooling force and actual beam parameters are not considered in detail. In this section, we simulate the cooling process using actual beam parameters and study the dependence of the cooling rate on dispersion.

The beam parameters in simulation are listed in Table I. This electron beam setting is specially designed for the EIC project at BNL [18,19], in which the electron beam with the energy of $150 \mathrm{MeV}$ is used to cool the $275 \mathrm{GeV}$ proton beam. At such high energy, transverse cooling is much weaker than longitudinal cooling. Therefore, dispersive electron cooling is desired in the design to balance the cooling rate in three dimensions. In Table I, we see that the rms velocity of the proton beam is less than that of the electron beam, which means most of protons are in the linear cooling force region. In addition, the beta function in the cooling section is pretty large, so the phase advance is small and beam distribution will not change too much along the $170 \mathrm{~m}$ cooling section. Based on these parameters, we believe that the analytical model can be used here to estimate the dispersive cooling rates, and it can also be compared with the simulation.

In the simulation, we use the multiparticle tracking code TRACKIT, which has been benchmarked with the experiment [14]. In the code, the cooling force is fully calculated by the Eq. (2) in Ref. [14] without any approximation. Particularly, the electron beam distribution with dispersion [Eqs. (17) and (18)] is applied in this simulation, and the cooling section is cut into three slices to include the betatron motion of electron beam. Since we only focus on the cooling rate, other effects such as IBS and space charge are not considered during tracking. The cooling rates are calculated based on the tracking result of the cooling process. It is worth noting that the transverse cooling rate corresponds to the beam emittance while longitudinal rate to the momentum amplitude, as defined in Eq. (12). The dependence of the cooling rate in three dimensions on electron dispersion $D_{e}$ and ion dispersion $D_{i}$ is shown in Fig. 4. We see that due to the electron and ion dispersion, the horizontal cooling rate is increased, while the longitudinal and vertical cooling rates are reduced, which is consistent with the conclusion of the analytical model.

According to the simulation without dispersion, we estimate the value of $C_{p} / C_{x}$ is about 5.2. With this number, the analytical model is compared with the simulation, as shown in Fig. 5. It shows that the analytical model is in good agreement with the simulation. The discrepancy in Fig. 5 is mainly due to the nonlinear terms of the cooling force, from which we see that the nonlinear cooling force reenforces the redistribution effect.
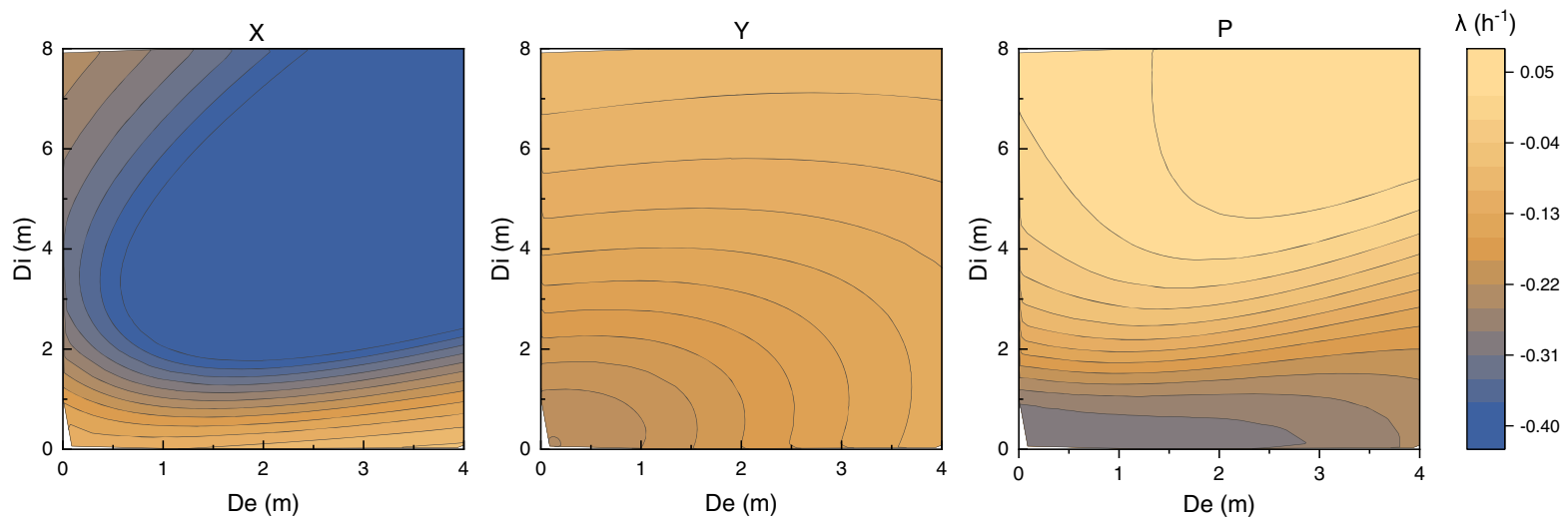

FIG. 4. Simulation results of the dependence of cooling rate on dispersion. 

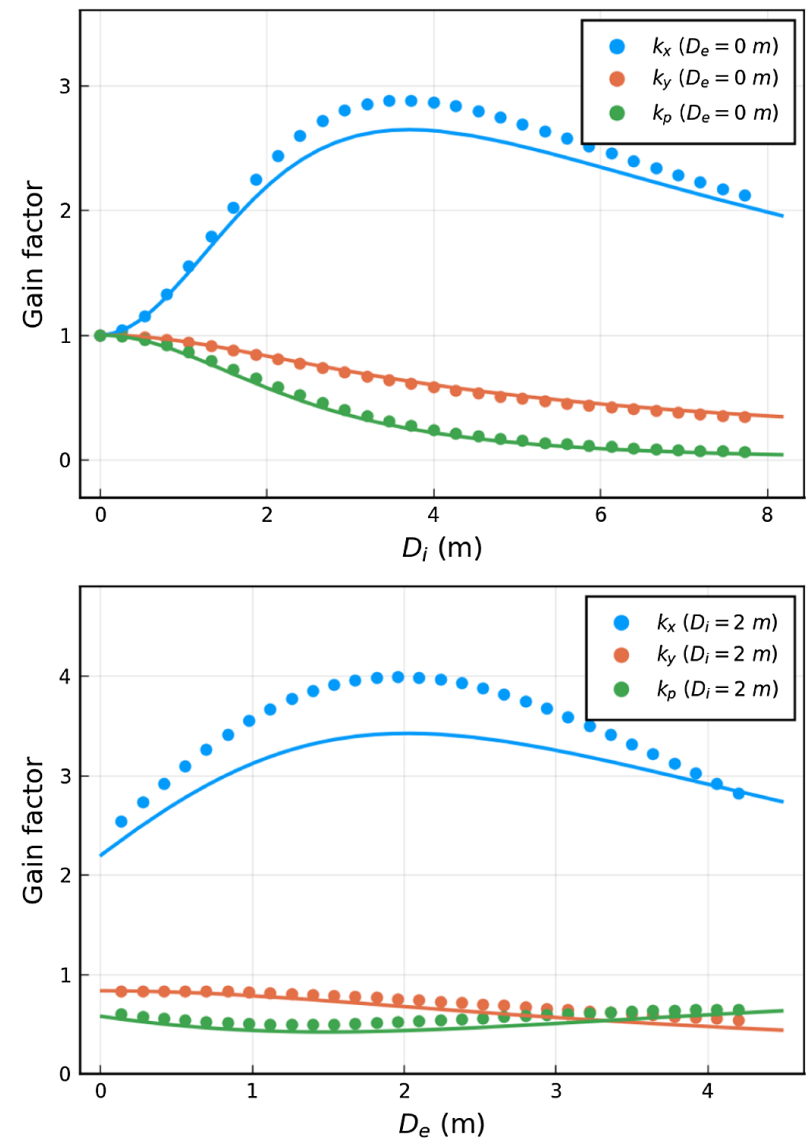

FIG. 5. Comparison between the simulation (dots) and analytical model (solid line) of the gain factor with $C_{p} / C_{x}=5.2$.

\section{SUMMARY}

We present a analytical model to explain and estimate the redistribution effect of dispersion on the transverse and longitudinal cooling rates. It demonstrates that the coupling effect in dispersive electron cooling can redistribute the cooling rates between the horizontal and longitudinal directions. Although the change of beam density caused by dispersion will reduce the cooling rates in all three dimensions, the horizontal cooling rate still can be enhanced by the coupling effect, as long as the dispersion is not too large. This redistribution effect benefits from both ion dispersion and electron dispersion, and is more significant when there is a large difference between the horizontal and longitudinal cooling rates, as occurs for high-energy beams. As a result, the dispersive cooling would be of particular value for high-energy electron cooling in the future.

\section{ACKNOWLEDGMENTS}

The authors would like to thank A. Fedotov, J. Kewisch, and D. Kayran for useful suggestions and discussions. This work is supported by the U.S. Department of Energy.

\section{APPENDIX: CALCULATION OF THE COOLING RATE}

In this section, we only give the details of the derivation on the horizontal cooling rate. Based on the Eq. (7), the mean values of the emittance change for the first order is

$$
\left\langle\Delta \epsilon_{x 1}\right\rangle=-C \beta_{x}\left\langle n_{e} x^{\prime 2}\right\rangle+\frac{C D_{i}}{\beta_{x}}\left\langle n_{e} \delta x_{\beta}\right\rangle .
$$

Since $n_{e}\left(x_{\beta}+D_{i} \delta, y_{\beta}, s\right)$ and $x^{\prime}$ are irrelevant, $\left\langle n_{e} x^{\prime 2}\right\rangle=$ $\left\langle n_{e}\right\rangle\left\langle x^{\prime 2}\right\rangle$. We know that $\beta_{x}\left\langle x^{\prime 2}\right\rangle=\epsilon_{x, \text { rms }}$ and these variables $\left(x_{\beta}, y_{\beta}, s, x^{\prime}, y^{\prime}\right.$, and $\left.\delta\right)$ are independent of each other, the value of $\left\langle n_{e}\right\rangle$ can be calculated by

$$
\begin{aligned}
\left\langle n_{e}\right\rangle= & M \int_{-\infty}^{\infty} \exp \left[-\frac{\left(x_{\beta}+D_{i} \delta\right)^{2}}{2 \sigma_{e x}^{2}}-\frac{y_{\beta}^{2}}{2 \sigma_{e y}^{2}}-\frac{s^{2}}{2 \sigma_{e s}^{2}}\right] \\
& \times \exp \left[-\frac{x_{\beta}^{2}}{2 \sigma_{i x}^{2}}-\frac{y_{\beta}^{2}}{2 \sigma_{i y}^{2}}-\frac{s^{2}}{2 \sigma_{i s}^{2}}-\frac{\delta^{2}}{2 \delta_{i p}^{2}}\right] d x_{\beta} d y_{\beta} d s d \delta \\
= & M \int_{-\infty}^{\infty} \exp \left(-\frac{y_{\beta}^{2}}{2 \sigma_{e y}^{2}}-\frac{y_{\beta}^{2}}{2 \sigma_{i y}^{2}}\right) d y_{\beta} . \\
& \times \int_{-\infty}^{\infty} \exp \left(-\frac{s^{2}}{2 \sigma_{e s}^{2}}-\frac{s^{2}}{2 \sigma_{i s}^{2}}\right) d s . \\
& \times \int_{-\infty}^{\infty} \exp \left[-\frac{\left(x_{\beta}+D_{i} \delta\right)^{2}}{2 \sigma_{e x}^{2}}-\frac{x_{\beta}^{2}}{2 \sigma_{i x}^{2}}-\frac{\delta^{2}}{2 \delta_{i p}^{2}}\right] d x_{\beta} d \delta,
\end{aligned}
$$

where

$$
M=\frac{N_{e 0}}{(2 \pi)^{7 / 2} \sigma_{e x} \sigma_{e y} \sigma_{e s} \sigma_{i x} \sigma_{i y} \sigma_{i s} \delta_{i p}} ;
$$

also

$$
\begin{aligned}
\left\langle n_{e} \delta x_{\beta}\right\rangle= & M \int_{-\infty}^{\infty} \exp \left(-\frac{y_{\beta}^{2}}{2 \sigma_{e y}^{2}}-\frac{y_{\beta}^{2}}{2 \sigma_{i y}^{2}}\right) d y_{\beta} . \\
& \times \int_{-\infty}^{\infty} \exp \left(-\frac{s^{2}}{2 \sigma_{e s}^{2}}-\frac{s^{2}}{2 \sigma_{i s}^{2}}\right) d s . \\
& \times \int_{-\infty}^{\infty} \delta x_{\beta} \exp \left[-\frac{\left(x_{\beta}+D_{i} \delta\right)^{2}}{2 \sigma_{e x}^{2}}-\frac{x_{\beta}^{2}}{2 \sigma_{i x}^{2}}-\frac{\delta^{2}}{2 \delta_{i p}^{2}}\right] d x_{\beta} d \delta .
\end{aligned}
$$

Base on the solutions in Ref. [20], the integrals are easy to calculate 


$$
\begin{aligned}
I_{1} & =\int_{-\infty}^{\infty} \exp \left(-\frac{y_{\beta}^{2}}{2 \sigma_{e y}^{2}}-\frac{y_{\beta}^{2}}{2 \sigma_{i y}^{2}}\right) d y_{\beta}=\frac{\sqrt{2 \pi} \sigma_{e y} \sigma_{i y}}{\sqrt{\sigma_{e y}^{2}+\sigma_{i y}^{2}}}, \\
I_{2} & =\int_{-\infty}^{\infty} \exp \left(-\frac{s^{2}}{2 \sigma_{e s}^{2}}-\frac{s^{2}}{2 \sigma_{i s}^{2}}\right) d s=\frac{\sqrt{2 \pi} \sigma_{e s} \sigma_{i s}}{\sqrt{\sigma_{e s}^{2}+\sigma_{i s}^{2}}}, \\
I_{3} & =\int_{-\infty}^{\infty} \exp \left[-\frac{\left(x_{\beta}+D_{i} \delta\right)^{2}}{2 \sigma_{e x}^{2}}-\frac{x_{\beta}^{2}}{2 \sigma_{i x}^{2}}-\frac{\delta^{2}}{2 \delta_{i p}^{2}}\right] d x_{\beta} d \delta \\
& =\frac{\pi}{\sqrt{a b-c^{2}}}, \quad \text { and } \\
I_{4} & =\int_{-\infty}^{\infty} \delta x_{\beta} \exp \left[-\frac{\left(x_{\beta}+D_{i} \delta\right)^{2}}{2 \sigma_{e x}^{2}}-\frac{x_{\beta}^{2}}{2 \sigma_{i x}^{2}}-\frac{\delta^{2}}{2 \delta_{i p}^{2}}\right] d x_{\beta} d \delta \\
& =\frac{\pi c}{2 \sqrt{\left(a b-c^{2}\right)^{3}}},
\end{aligned}
$$

where $a=\frac{1}{2 \sigma_{e x}^{2}}+\frac{1}{2 \sigma_{i x}^{2}}, b=\frac{D_{i}^{2}}{2 \sigma_{e x}^{2}}+\frac{1}{2 \delta_{i p}^{2}}$, and $c=-\frac{D_{i}}{2 \sigma_{e x}^{2}}$. Then,

$$
\begin{aligned}
\left\langle n_{e}\right\rangle & =M \frac{\sqrt{2 \pi} \sigma_{e y} \sigma_{i y}}{\sqrt{\sigma_{e y}^{2}+\sigma_{i y}^{2}}} \frac{\sqrt{2 \pi} \sigma_{e s} \sigma_{i s}}{\sqrt{\sigma_{e s}^{2}+\sigma_{i s}^{2}}} \frac{\pi}{\sqrt{a b-c^{2}}} \text { and } \\
\left\langle n_{e} \delta x_{\beta}\right\rangle & =M \frac{\sqrt{2 \pi} \sigma_{e y} \sigma_{i y}}{\sqrt{\sigma_{e y}^{2}+\sigma_{i y}^{2}}} \frac{\sqrt{2 \pi} \sigma_{e s} \sigma_{i s}}{\sqrt{\sigma_{e s}^{2}+\sigma_{i s}^{2}}} \frac{\pi c}{2 \sqrt{\left(a b-c^{2}\right)^{3}}} .
\end{aligned}
$$

And the final form of $\left\langle\Delta \epsilon_{x 1}\right\rangle$ is

$$
\begin{aligned}
\left\langle\Delta \epsilon_{x 1}\right\rangle & =-C \epsilon_{x, \mathrm{rms}}\left\langle n_{e}\right\rangle+\frac{C D_{i}}{\beta_{x}}\left\langle n_{e}\right\rangle \frac{c}{2\left(a b-c^{2}\right)} \quad \text { and } \\
& =-\hat{n}_{e 0} \epsilon_{x, \mathrm{rms}}\left(C+\frac{C D_{i}^{2} \delta_{i p}^{2}}{\sigma_{e x}^{2}+\sigma_{i x}^{2}+D_{i}^{2} \delta_{i p}^{2}}\right)
\end{aligned}
$$

where

$$
\hat{n}_{e 0}=\frac{(2 \pi)^{-3 / 2} N_{e 0}}{\sqrt{\left(\sigma_{e y}^{2}+\sigma_{i y}^{2}\right)\left(\sigma_{e s}^{2}+\sigma_{i s}^{2}\right)\left(\sigma_{e x}^{2}+\sigma_{i x}^{2}+D_{i}^{2} \delta_{i p}^{2}\right)}} .
$$

On the other hand, the second order of emittance change in Eq. (7) is

$$
\left\langle\Delta \epsilon_{x 2}\right\rangle=\frac{C^{2} D_{i}^{2}}{2 \beta_{x}}\left\langle n_{e}^{2} \delta^{2}\right\rangle+\frac{C^{2} \beta_{x}}{2}\left\langle n_{e}^{2}\right\rangle\left\langle x^{\prime 2}\right\rangle
$$

Using the same method, we obtain

$$
\left\langle\Delta \epsilon_{x 2}\right\rangle=C^{2} \hat{n}_{e 1}\left[\frac{\epsilon_{x, \mathrm{rms}}}{2}+\frac{D_{i}^{2} \delta_{i p}^{2}\left(\sigma_{e x}^{2}+2 \sigma_{i x}^{2}\right)}{2 \beta_{x}\left(\sigma_{e x}^{2}+2 \sigma_{i x}^{2}+2 D_{i}^{2} \delta_{i p}^{2}\right)}\right],
$$

where

$$
\hat{n}_{e 1}=\frac{(2 \pi)^{-3} N_{e 0}^{2}\left(\sigma_{e x} \sigma_{e y} \sigma_{e s}\right)^{-1}}{\sqrt{\left(\sigma_{e y}^{2}+2 \sigma_{i y}^{2}\right)\left(\sigma_{e s}^{2}+2 \sigma_{i s}^{2}\right)\left(\sigma_{e x}^{2}+2 \sigma_{i x}^{2}+2 D_{i}^{2} \delta_{i p}^{2}\right)}} .
$$

Comparing Eqs. (A7) and (A9), we get

$$
\left|\frac{\left\langle\Delta \epsilon_{x 2}\right\rangle}{\left\langle\Delta \epsilon_{x 1}\right\rangle}\right| \simeq \frac{C N_{e 0}}{(2 \pi)^{3 / 2} \sigma_{e x} \sigma_{e y} \sigma_{e s}}=C n_{e 0},
$$

where $n_{e 0}$ is the maximum density of electron beam. We know $C n_{e 0}=\left|\frac{\Delta u_{i}}{u_{i}}\right|_{\max }$ is the maximum cooling gradient, which is very small (roughly less than $10^{-3}$ ). So we conclude that the contribution of the high order term in Eq. (7) is such small that can be ignored, and we can finally obtain Eq. (12). The calculation in longitudinal and vertical planes are the same.

[1] G. I. Budker, An effective method of damping particle oscillations in proton and antiproton storage rings, Sov. At. Energy 22, 438 (1967).

[2] H. Poth, Applications of electron cooling in atomic, nuclear and high-energy physics, Nature (London) 345, 399 (1990).

[3] C. Montag, G. Bassi, J. Beebe-Wang, J. S. Berg, M. Blaskiewicz, A. Blednykh, J. Brennan, S. Brooks, K. Brown, K. Drees et al., eRHIC design overview, in Proceedings of the 10th International Particle Accelerator Conference (IPAC'19), Melbourne, Australia, 2019 (JACoW Publishing, Geneva, Switzerland, 2019), pp. 45-48.

[4] D. P. Anderle, V. Bertone, X. Cao, L. Chang, N. Chang, G. Chen, X. Chen, Z. Chen, Z. Cui, L. Dai et al., Electron-ion collider in China, Front. Phys. 16, 64701 (2021).

[5] S. Nagaitsev, D. Broemmelsiek, A. Burov, K. Carlson, C. Gattuso, M. Hu, T. Kroc, L. Prost, S. Pruss, M. Sutherland et al., Experimental Demonstration of Relativistic Electron Cooling, Phys. Rev. Lett. 96, 044801 (2006).

[6] A. Fedotov, Z. Altinbas, S. Belomestnykh, I. Ben-Zvi, M. Blaskiewicz, M. Brennan, D. Bruno, C. Brutus, M. Costanzo, A. Drees et al., Experimental Demonstration of Hadron Beam Cooling Using Radio-Frequency Accelerated Electron Bunches, Phys. Rev. Lett. 124, 084801 (2020).

[7] V. Parkhomchuk, New insights in the theory of electron cooling, Nucl. Instrum. Methods Phys. Res., Sect. A 441, 9 (2000).

[8] S. Van Der Meer, Stochastic cooling and the accumulation of antiprotons, Rev. Mod. Phys. 57, 689 (1985).

[9] D. Möhl, G. Petrucci, L. Thorndahl, and S. Van Der Meer, Physics and technique of stochastic cooling, Phys. Rep. 58, 73 (1980).

[10] I. Lauer, U. Eisenbarth, M. Grieser, R. Grimm, P. Lenisa, V. Luger, T. Schätz, U. Schramm, D. Schwalm, and M. Weidemüller, Transverse Laser Cooling of a Fast Stored Ion Beam through Dispersive Coupling, Phys. Rev. Lett. 81, 2052 (1998). 
[11] J. Bosser, C. Carli, M. Chanel, L. Marie, D. Möhl, and G. Tranquille, On the optimum dispersion of a storage ring for electron cooling with high space charge, Nucl. Instrum. Methods Phys. Res., Sect. A 441, 60 (2000).

[12] M. Beutelspacher, M. Grieser, K. Noda, D. Schwalm, and A. Wolf, Dispersive electron cooling experiments at the heavy ion storage ring TSR, Nucl. Instrum. Methods Phys. Res., Sect. A 512, 459 (2003).

[13] Y.S. Derbenev, Theory of electron cooling, arXiv:1703. 09735.

[14] H. Zhao, M. Blaskiewicz, A. Fedotov, W. Fischer, X. Gu, D. Kayran, J. Kewisch, C. Liu, S. Seletskiy, V. Schoefer et al., Cooling simulation and experimental benchmarking for an rf-based electron cooler, Phys. Rev. Accel. Beams 23, 074201 (2020).

[15] T. Schramm, Realistic elliptical potential wells for gravitational lens models, Astron. Astrophys. 231, 19 (1990), http:// legacy.adsabs.harvard.edu/pdf/1990A\&A...231...19S.
[16] H. Pishro-Nik, Introduction to probability, statistics, and random processes, Kappa Research LLC, 2014, https:// www.probabilitycourse.com.

[17] M. Blaskiewicz, Dispersion and electron cooling (Brookhaven National Laboratory (BNL), Upton, NY (United States), 2019) Technical Report No. BNL-210932-2019TECH.

[18] H. Zhao, J. Kewisch, M. Blaskiewicz, and A. Fedotov, Ring-based electron cooler for high energy beam cooling, Phys. Rev. Accel. Beams 24, 043501 (2021).

[19] J. Kewisch, H. Zhao, M. Blaskiewicz, and A. Fedotov, Design of electron storage ring for high-energy EIC cooling (Brookhaven National Laboratory (BNL), Upton, NY (United States), 2020) Technical Report No. BNL-2207322020-TECH.

[20] A. Jeffrey and H. H. Dai, Handbook of Mathematical Formulas and Integrals (Elsevier, New York, 2008). 